\title{
Safety of biliary stent placement followed by definitive chemoradiotherapy in patients with pancreatic cancer with bile duct obstruction
}

\author{
Sara Hayakawa ${ }^{1}$, Kei Ito ${ }^{1}$, Jin Hayakawa ${ }^{2}$, Keiko Nemoto Murofushi ${ }^{1}$, Katsuyuki Karasawa ${ }^{1}$ \\ ${ }^{1}$ Division of Radiation Oncology, Department of Radiology, Tokyo Metropolitan Cancer and Infectious Diseases Center Komagome Hospital, \\ Tokyo, Japan; ${ }^{2}$ Department of Medical Informatics and Economics, Graduate School of Medicine, the University of Tokyo, Tokyo, Japan \\ Contributions: (I) Conception and design: S Hayakawa, K Ito; (II) Administrative support: S Hayakawa; (III) Provision of study materials or patients: \\ S Hayakawa; (IV) Collection and assembly of data: S Hayakawa; (V) Data analysis and interpretation: S Hayakawa, J Hayakawa; (VI) Manuscript \\ writing: All authors; (VII) Final approval of manuscript: All authors. \\ Correspondence to: Kei Ito. Division of Radiation Oncology, Department of Radiology, Tokyo Metropolitan Cancer and Infectious Diseases Center \\ Komagome Hospital, 3-18-22 Honkomagome, Bunkyo-ku, Tokyo 113-8677, Japan. Email: keiito@cick.jp.
}

Background: Although patients with malignant bile duct obstruction due to pancreatic cancer are often
initially treated with biliary stent placement, concurrent chemoradiotherapy with stents poses a potential risk
of increased toxicity. This retrospective study aimed to evaluate the safety of biliary stent placement followed
by definitive concurrent chemoradiotherapy in patients with pancreatic cancer.
Methods: Patients with pancreatic cancer who underwent either a plastic stent or a self-expanding metallic
stent placement for malignant bile duct obstruction before definitive concurrent chemoradiotherapy were
retrospectively reviewed. Radiotherapy was delivered in 1.8 Gy per fraction to a total dose of 50.4 Gy.
Gemcitabine, TS-1 plus Gemcitabine, or TS-1 was the concurrent chemotherapy/regimen. The primary
endpoint was the rate of biliary stent-related toxicities, defined as biliary bleeding, duodenal perforation, or
bile duct perforation.

Results: Thirty patients were included. Plastic stents were placed in 23 patients and self-expanding metallic stent in seven patients at the start of irradiation. The median follow-up time was 20 (range, 2-63) months, and 27 patients (90\%) completed concurrent chemoradiotherapy. Biliary stent-related toxicity (grade 3 biliary bleeding) was confirmed in one patient (3\%) with a plastic stent 9 months after concurrent chemoradiotherapy. The median duration of locoregional control, progression-free survival, and overall survival were $31.1,7.3$, and 10.5 months, respectively.

Conclusions: Stent placement followed by concurrent chemoradiotherapy was not associated with an apparent increase in toxicity and may be an appropriate treatment for patients with locally advanced pancreatic head cancer with bile duct obstruction.

Keywords: Pancreatic cancer; bile duct obstruction; biliary stent; chemoradiotherapy

Submitted Apr 11, 2021. Accepted for publication Aug 23, 2021.

doi: 10.21037/jgo-21-198

View this article at: https://dx.doi.org/10.21037/jgo-21-198

\section{Introduction}

Approximately $70 \%$ of patients with pancreatic cancer have malignant bile duct obstruction at diagnosis (1), which is correlated with reduced quality of life and survival times (2). The standard-of-care management of obstructive jaundice is drainage, and the most common drainage method is endoscopic biliary stent placement using a plastic stent (PS) or self-expanding metallic stent (SEMS) (1).

Although definitive concurrent chemoradiotherapy (CCRT) is one of the standard treatments for pancreatic 
cancer (3), only a few reports on the safety of CCRT following biliary stent placement have been published. Some case reports of biliary bleeding following combined radiotherapy and biliary stent placement have also been published $(4,5)$, and histopathological findings at autopsy after combined radiotherapy and biliary stenting for cholangiocarcinoma have shown inflammatory cell infiltration and fibrosis in the subepithelium of the bile duct (6). The clinical practice guidelines for pancreatic cancer in Japan have highlighted the lack of safety data on radiotherapy (7), and some institutions do not administer irradiation to patients with biliary stents. Therefore, CCRT for patients with locally advanced pancreatic cancer with a biliary stent may carry an additional risk of biliary bleeding, duodenal perforation, and bile duct perforation, which has not yet been clinically evaluated. The safety and tolerability of CCRT for patients with pancreatic cancer with biliary stents must be explored to allow for optimal treatment selection.

The present study aimed to evaluate the safety of biliary stent placement followed by definitive CCRT in patients with pancreatic cancer.

We present the following article in accordance with the STROBE reporting checklist (available at https://dx.doi. org/10.21037/jgo-21-198).

\section{Methods}

\section{Patients}

This retrospective review included patients with pancreatic cancer who underwent biliary stent (PS or SEMS) placement for malignant bile duct obstruction followed by definitive CCRT at a single Japanese institution (Komagome Hospital, Tokyo, Japan) between May 2012 and June 2019. Patients were included if they met the following criteria: (I) pathologically or clinically diagnosed pancreatic cancer, (II) a locally advanced unresectable tumor classified as stage III or stage IV only if the metastatic disease was limited to the single para-aortic lymph node, judged based on the TNM classification (8) or was inoperable due to a medical situation (e.g., old age or comorbidity), (III) treatment with definitive CCRT, and (IV) a biliary stent placed before initiating CCRT. Patients treated with chemotherapy before CCRT or conversion surgery after CCRT were excluded. A tumor with a contact of $>180^{\circ}$ with the superior mesenteric or celiac artery, a tumor contacting the celiac artery and with aortic involvement, or an unreconstructible superior mesenteric or portal vein due to tumor involvement or occlusion was considered unresectable according to the National Comprehensive Cancer Network guidelines (9).

\section{Ethical statement}

The study was conducted in accordance with the Declaration of Helsinki (as revised in 2013). This study was approved by the institutional ethical review board of Tokyo Metropolitan Cancer and Infectious Diseases Center Komagome Hospital (approval number: 2359), and informed consent was obtained in the form of an opt-out option displayed on the website.

\section{Biliary stent}

All patients had a biliary stent placed at the time of their initial jaundice treatment; a PS was preferred at this point. The type, length, and caliber of the stent were determined at the primary endoscopist's discretion. Planned routine stent exchange after several weeks or months to prevent stent occlusion was not performed.

\section{Radiotherapy}

Patients underwent contrast-enhanced computed tomography (CT) simulation and four-dimensional CT or radioscopy to assess respiratory motion. The gross tumor volume (GTV) was defined as including the primary tumor and any involved nodes. The internal margin was added to the GTV based on four-dimensional CT or radioscopy according to respiratory motion (defined as the total GTV). The clinical target volume (CTV) comprised the total GTV plus a uniform 5 -mm margin and peri-celiac and perimesenteric artery lymph nodes as prophylactic lymph node areas. A 5-mm margin was added to the CTV to create the planning target volume. All biliary stents were partially included in the GTV.

External beam radiation therapy was administered using three-dimensional conformal radiation therapy, typically with four ports or intensity-modulated radiation therapy using photon beams with energy $\geq 6-\mathrm{MV}$. A total dose of 50.4 Gy was delivered in 28 fractions of 1.8 Gy, 5 days per week, for 5.5 weeks. A typical target delineation and dose distribution are shown in Figure 1. Dose constraints of the surrounding risk organs such as the duodenum or bile duct were not set. No density override was performed for SEMS artifacts on planning CT. Radiotherapy was started after the 

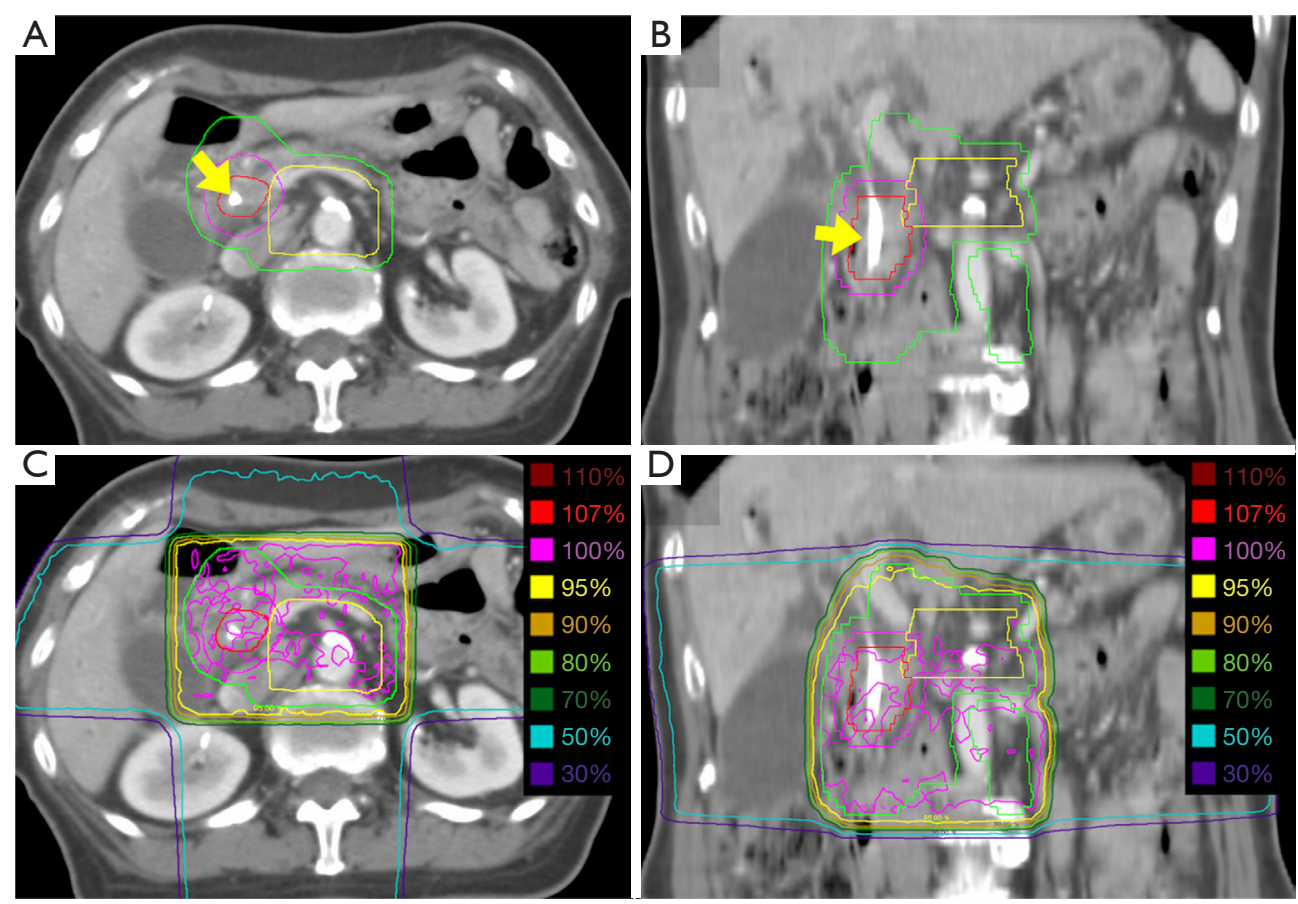

Figure 1 Images from a representative case. Axial (A) and coronal (B) contrast-enhanced CT images with contouring for planning radiotherapy [gross tumor volume (red), clinical target volume (pink, yellow), planning target volume (green), and plastic stent (yellow arrow)]. Axial (C) and coronal (D) contrast-enhanced CT images with dose distribution. CT, computed tomography.

serum total bilirubin level decreased to $<3 \mathrm{mg} / \mathrm{dL}$.

\section{Chemotherapy}

Patients received one of the following chemotherapies: (I) gemcitabine (GEM) $\left(1,000 \mathrm{mg} / \mathrm{m}^{2}\right.$ administered intravenously once per week for 3 weeks) alone, (II) GEM $\left(200 \mathrm{mg} / \mathrm{m}^{2}\right.$ administered intravenously once per week for 6 weeks) plus TS-1 (80 mg/m ${ }^{2}$ administered orally twice daily after breakfast and dinner on the day of irradiation), or (III) TS-1 $\left(80 \mathrm{mg} / \mathrm{m}^{2}\right.$ administered orally twice daily after breakfast and dinner on the day of irradiation) alone. Our treatment policy was to continue maintenance chemotherapy for patients with a partial response to stable disease after CCRT.

\section{Evaluation}

The primary endpoint of this study was the biliary stentrelated toxicity rate. Biliary stent-related toxicities were defined as biliary bleeding, duodenal perforation, or bile duct perforation. The secondary endpoints were other adverse events (AEs), treatment compliance, initial response rate, locoregional control (LC), progression-free survival (PFS), and overall survival (OS). An acute AE was defined as an $\mathrm{AE}$ that occurred within 90 days after CCRT, and a late $\mathrm{AE}$ was defined as an $\mathrm{AE}$ that occurred $>90$ days after CCRT. AEs were evaluated at monthly follow-up visits according to the National Cancer Institute Common Terminology Criteria for Adverse Events version 5.0 (10). Treatment compliance was analyzed based on breaks in radiotherapy recorded in days and the percentage of CCRT discontinuation. Evaluations of the initial tumor response were performed using CT and magnetic resonance imaging at 1- or 2-month intervals after completing CCRT according to the Response Evaluation Criteria in Solid Tumours guidelines (11). LC was calculated from the start of radiotherapy to the date of the first documented local relapse. PFS was calculated from the start of radiotherapy to locoregional/distant failure or death. OS was defined as the time between radiotherapy initiation and the date of death from any cause.

\section{Statistical analysis}

The LC, PFS, and OS were estimated using the Kaplan- 
Table 1 Patient characteristics

\begin{tabular}{|c|c|c|}
\hline Characteristic & Variable & $\mathrm{N}[\%]$ \\
\hline \multirow[t]{2}{*}{ Sex } & Male & $17[57]$ \\
\hline & Female & $13[43]$ \\
\hline Age (years) & Median [range] & 71 [51-87] \\
\hline \multirow[t]{2}{*}{ Diabetes mellitus } & No & 19 [63] \\
\hline & Yes & $11[37]$ \\
\hline Primary site & Pancreatic head & $30[100]$ \\
\hline \multirow[t]{6}{*}{ Clinical stage } & IA & $1[3]$ \\
\hline & IB & $1[3]$ \\
\hline & $\| \mathrm{A}$ & $1[3]$ \\
\hline & IIB & $4[13]$ \\
\hline & III & $21[70]$ \\
\hline & IV & $2[7]$ \\
\hline \multirow[t]{3}{*}{ Resectability } & Resectable & $4[13]$ \\
\hline & Borderline-resectable & $2[7]$ \\
\hline & Unresectable & $24[80]$ \\
\hline \multirow[t]{3}{*}{ Chemotherapy } & TS-1 & $18[60]$ \\
\hline & GEM & $1[3]$ \\
\hline & GEM + TS-1 & $11[37]$ \\
\hline \multirow[t]{2}{*}{ Types of stent } & Plastic stent & $23[77]$ \\
\hline & Self-expanding metallic stent & 7 [23] \\
\hline \multirow{2}{*}{$\begin{array}{l}\text { Radiotherapy } \\
\text { modality }\end{array}$} & 3D-CRT & 28 [93] \\
\hline & IMRT & $2[7]$ \\
\hline
\end{tabular}

$\mathrm{n}=30$ patients. GEM, gemcitabine; 3D-CRT, three-dimensional conformal radiotherapy; IMRT, intensity-modulated radiation therapy.

Meier method. All statistical analyses were performed using R 3.6.3 (R Foundation for Statistical Computing, Vienna, Austria) and EZR version 1.51 (Saitama Medical Center) (12).

\section{Results}

\section{Patient characteristics}

Between May 2012 and June 2019, 276 patients were treated with preoperative or definitive radiotherapy for pancreatic cancer. Of these, 112 patients underwent biliary stent placement for malignant bile duct obstruction before CCRT. Sixty-two patients who underwent CCRT as neoadjuvant treatment, three patients who underwent chemotherapy before CCRT, and one patient who underwent conversion surgery after CCRT were excluded. The remaining 30 patients were included in this study. Patient and tumor characteristics are summarized in Table 1. The median age was 71 (range, 51-87) years. The type of stent placed at the start of irradiation was a PS in 23 patients and an SEMS in seven patients. Eleven patients had diabetes mellitus.

Twenty-seven patients (90\%) completed CCRT. Three patients discontinued CCRT due to cholangitis, endoscopic retrograde cholangiopancreatography-induced pancreatitis, and poorly controlled febrile neutropenia (one patient each). Among the five patients who underwent treatment breaks or CCRT discontinuation due to cholangitis, four patients had PS placement. Among the nine patients with treatment breaks or CCRT discontinuation due to hematologic toxicity, six patients were treated with a GEM plus TS-1 regimen.

\section{Toxicity}

The median follow-up time was 20 (range, 2-63) months. Among the 30 patients treated with CCRT, biliary stent-related toxicity was observed in one (3\%), who experienced biliary bleeding. This patient was a 71-yearold man who underwent PS placement. After confirming malignancy by endoscopic ultrasound-guided fineneedle aspiration biopsy, CCRT with the GEM plus TS-1 regimen was performed and completed. Initial evaluation with CT and magnetic resonance imaging revealed multiple liver and bone metastases, and the patient received GEM and nab-paclitaxel as systemic therapy. Nine months after starting CCRT, the patient developed anemia and bile duct stent obstruction. Red blood cell transfusion was performed, and the PS was replaced with an SEMS. At the time of replacement, blood outflow through the PS was observed, and endoscopic retrograde cholangiopancreatography revealed stenosis of the lower bile duct and the presence of a hematoma in the upstream common bile duct; however, the anemia had not worsened. The patient continued to experience melena and developed cardiac arrest due to massive hemorrhage 5 days after SEMS replacement but was revived by life-saving measures. Subsequent CT revealed a pseudoaneurysm in the posterior superior pancreaticoduodenal artery immediately above the SEMS 



Figure 2 Images from a case of biliary bleeding after concurrent chemoradiotherapy. (A) Coronal CT images with dose distribution. (B) Coronal contrast-enhanced CT images of a pseudoaneurysm in the posterior superior pancreaticoduodenal artery immediately above the SEMS (yellow arrow). CT, computed tomography; SEMS, self-expanding metallic stent.

Table 2 Treatment toxicity

\begin{tabular}{|c|c|c|c|c|}
\hline \multirow{2}{*}{ Events } & \multicolumn{3}{|c|}{ Grade } & \multirow{2}{*}{ Total, n [\%] } \\
\hline & 2 & 3 & 4 & \\
\hline \multicolumn{5}{|l|}{ Acute adverse events } \\
\hline Cholangitis & 0 & 9 & 0 & $9[30]$ \\
\hline Anorexia & 1 & 6 & 0 & 7 [23] \\
\hline Nausea & 2 & 4 & 0 & $6[20]$ \\
\hline Diarrhea & 3 & 1 & 0 & $4[13]$ \\
\hline Biliary bleeding & 0 & 1 & 0 & $1[3]$ \\
\hline Liver abscess & 0 & 1 & 0 & $1[3]$ \\
\hline Gastritis & 1 & 0 & 0 & $1[3]$ \\
\hline Duodenal ulcer & 0 & 1 & 0 & $1[3]$ \\
\hline Leukopenia & 3 & 8 & 1 & $12[40]$ \\
\hline Febrile neutropenia & 0 & 1 & 0 & 1 [3] \\
\hline Anemia & 0 & 3 & 0 & $3[10]$ \\
\hline Thrombocytopenia & 3 & 2 & 0 & $5[16]$ \\
\hline \multicolumn{5}{|l|}{ Late adverse events } \\
\hline Cholangitis & 0 & 4 & 0 & $4[13]$ \\
\hline Gastric hemorrhage & 1 & 2 & 0 & $3[10]$ \\
\hline Duodenal ulcer & 1 & 0 & 0 & $1[3]$ \\
\hline Biliary bleeding & 0 & $1^{\dagger}$ & 0 & $1[3]$ \\
\hline
\end{tabular}

${ }^{\dagger}$, stent-related toxicity.

(Figure 2) with worsening of the primary disease. Thus, interventional radiology was performed to embolize the aneurysm using n-butyl-2-cyanoacrylate. The patient survived without rebleeding but died 10 months after CCRT due to primary disease progression.

The most common acute non-hematological AE was cholangitis, which was observed in nine $(30 \%)$ patients. Of these nine patients, eight (89\%) had a PS, and one $(11 \%)$ had an SEMS. The second most common nonhematological acute AEs were gastrointestinal toxicities, including anorexia and nausea, which were observed as grade 2 and $3 \mathrm{AEs}$ in $7(23 \%)$ and $6(20 \%)$ patients, respectively. However, these AEs were manageable. A patient who developed biliary bleeding as an acute grade 4 $\mathrm{AE}$ was considered not to have stent-related toxicity because he had liver metastases from renal cancer, and interventional radiology showed bleeding from liver metastases. No acute grade $5 \mathrm{AEs}$ were observed. The most common late $\mathrm{AE}$ was cholangitis, which was observed in four $(13 \%)$ patients, one of whom experienced biliary bleeding when the stent was replaced. Gastric hemorrhage and duodenal ulcer occurred in three $(10 \%)$ and one $(3 \%)$ patient(s), respectively. No grade 4 or higher late AEs were encountered. Table 2 shows the acute and late AEs.

Treatment breaks in radiotherapy were noted in 14 patients (47\%), and the median break duration was 6 days (range, 1-11 days). The reasons for radiotherapy breaks were cholangitis in 4 patients, grade 3 hematologic toxicity in seven patients, grade 3 appetite loss in 1 patient, grade 3 hematologic toxicity and duodenal ulcer in one patient, and duodenal stricture due to direct tumor invasion in one patient. In the four patients who developed cholangitis, the mean period between initial stent deployment to the onset of cholangitis was 35 (range, 14-47) days; treatment was completed after stent replacement. 


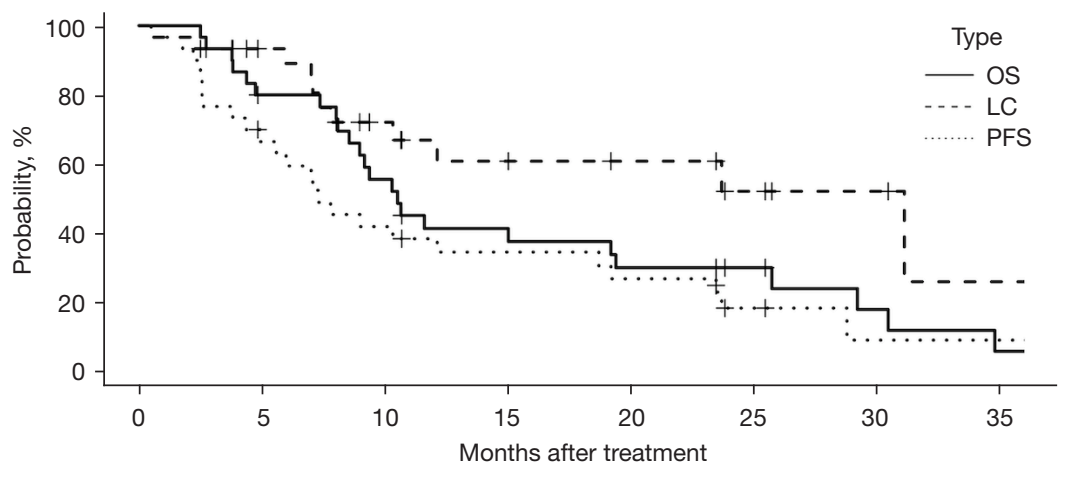

\begin{tabular}{|c|c|c|c|c|c|c|c|c|}
\hline \multicolumn{9}{|c|}{ Number at risk } \\
\hline OS & 30 & 23 & 16 & 11 & 8 & 6 & 3 & 1 \\
\hline LC & 30 & 22 & 14 & 10 & 8 & 5 & 3 & 1 \\
\hline PFS & 30 & 19 & 12 & 9 & 7 & 3 & 1 & 1 \\
\hline
\end{tabular}

Figure 3 Kaplan-Meier estimates of LC, PFS, and OS. LC, locoregional control; PFS, progression-free survival; OS, overall survival.

\section{Efficacy}

The initial responses were partial responses in $4(13 \%)$, stable disease in $20(67 \%)$, and progressive disease in 3 (10\%) patients; no patients showed complete response, and the data from three were unavailable. Maintenance chemotherapy was continued in $19(79 \%)$ patients among those showing partial response and stable disease. Recurrence was observed in 20 patients $(67 \%)$; of these, 6 patients experienced locoregional recurrence in the pancreas, 11 experienced distant metastases alone, and three experienced both locoregional recurrence and distant metastases. The 1 -year LC rate was $67 \%$, with a median LC of 31.1 months; the 1-year PFS rate was $39 \%$, with a median PFS of 7.3 months; and the 1-year OS rate was $41 \%$, with a median survival time of 10.5 months (Figure 3).

\section{Discussion}

The present study aimed to determine the safety and tolerability of CCRT in patients with locally advanced pancreatic head cancer who had undergone stent placement for malignant bile duct obstruction. The results revealed only one case of biliary bleeding and no apparent increase in the frequency or severity of toxicity.

Obstructive jaundice caused by pancreatic head cancer requires appropriate treatment as it worsens prognosis and lessens the quality of life. Stent insertion is a commonly used symptomatic treatment to relieve jaundice (1). As causal treatments, CCRT or chemotherapy are the established standard therapies for locally advanced unresectable pancreatic cancer (3). Some clinical data suggest that CCRT is superior to chemotherapy for local control (13) and biliary stent patency (14); hence, CCRT may be more appropriate than chemotherapy to prevent and relieve bile duct obstruction. Therefore, a combination of stent placement and CCRT is the most promising approach for malignant bile duct obstruction.

Several case reports on biliary bleeding after stent placement for malignant biliary obstruction have been published regardless of whether radiotherapy was performed (4,5,15-18). Some possible explanations of bleeding that have been reported are adhesion of the stent to the tumor, duodenal ulceration by the distal end of the stent, and mechanical irritation of the adjacent arterial wall by the stent (19). A large retrospective case series reported an arterial bleeding frequency of $1.0 \%$ (19/1,858 patients) after SEMS placement, and 10 of the 19 patients had a history of radiotherapy; the authors suggested that radiotherapy contributes to the formation of an aneurysm (19). However, the timing of stent placement, the overall population receiving radiotherapy, and details of radiotherapy, including the prescribed dose and irradiation fields, were not described; hence, the contribution of radiotherapy to bleeding remains unknown. The present study included only patients treated with a uniform dose fraction schedule and a unified treatment sequence of stent placement before CCRT. We found no evidence supporting the significant contribution of radiotherapy to enhanced toxicity during long-term follow-up (median follow-up, 20 months). We consider that CCRT is an acceptable treatment option in 
patients with biliary stents.

Concerning the type of stent for pancreatic head cancer with obstructive jaundice, a randomized controlled trial (20) comparing PS and SEMS in patients with nonresection pancreatic head cancer reported that SEMS had longer patency and a significantly lower frequency of stent occlusion than PS. In the present study, treatment breaks or discontinuation due to cholangitis was noted in five patients, four of whom had a PS. SEMS may be the preferred choice for the completion of CCRT.

Our study has several limitations. First, the sample size was small; hence, it was insufficient to determine conclusive results. Second, PS and SEMS were not assessed separately, which is necessary, as PS and SEMS differ in diameter and material. Notably, basic data have shown that SEMS enhance the radiation dose around the stent (21). The number of cases and events in the present study was too small to perform a comparative analysis. Third, death without biliary stent-related toxicity is a competing risk for toxicity assessments. However, these results follow the real-world setting because of the short survival time in patients with locally advanced pancreatic cancer. Finally, the histological changes caused by the combination of CCRT and stent placement were not evaluated pathologically. In the future, we plan to evaluate pathological changes in patients with or without stents who received CCRT as a preoperative treatment for pancreatic cancer.

\section{Conclusions}

In conclusion, patients who underwent biliary stent placement followed by CCRT had high compliance rates, which led to the completion of the treatment. In addition, there was no apparent increase in stent-related toxicity caused by CCRT. These findings suggest that the combination treatment is an optimal treatment strategy in patients with pancreatic cancer with bile duct obstruction.

\section{Acknowledgments}

Funding: This work was supported by the Clinical Research Fund of Tokyo Metropolitan Government to S Hayakawa (Grant Number R020303007).

\section{Footnote}

Reporting Checklist: The authors have completed the STROBE reporting checklist. Available at https://dx.doi. org/10.21037/jgo-21-198

Data Sharing Statement: Available at https://dx.doi. org/10.21037/jgo-21-198

Conflicts of Interest: All authors have completed the ICMJE uniform disclosure form (available at https://dx.doi. org/10.21037/jgo-21-198). The authors have no conflicts of interest to declare.

Ethical Statement: The authors are accountable for all aspects of the work in ensuring that questions related to the accuracy or integrity of any part of the work are appropriately investigated and resolved. The study was conducted in accordance with the Declaration of Helsinki (as revised in 2013). This study was approved by the institutional ethical review board of Tokyo Metropolitan Cancer and Infectious Diseases Center Komagome Hospital (approval number: 2359), and informed consent was obtained in the form of an opt-out option displayed on the website.

Open Access Statement: This is an Open Access article distributed in accordance with the Creative Commons Attribution-NonCommercial-NoDerivs 4.0 International License (CC BY-NC-ND 4.0), which permits the noncommercial replication and distribution of the article with the strict proviso that no changes or edits are made and the original work is properly cited (including links to both the formal publication through the relevant DOI and the license). See: https://creativecommons.org/licenses/by-nc-nd/4.0/.

\section{References}

1. Boulay BR, Parepally M. Managing malignant biliary obstruction in pancreas cancer: choosing the appropriate strategy. World J Gastroenterol 2014;20:9345-53.

2. Kruse EJ. Palliation in pancreatic cancer. Surg Clin North Am 2010;90:355-64.

3. Loehrer PJ Sr, Feng Y, Cardenes H, et al. Gemcitabine alone versus gemcitabine plus radiotherapy in patients with locally advanced pancreatic cancer: an Eastern Cooperative Oncology Group trial. J Clin Oncol 2011;29:4105-12.

4. Raja A, Papadopoulou A, Gillmore R. Pseudoaneurysm development after stenting for malignant biliary obstruction. Dig Liver Dis 2015;47:726.

5. Nezu Y, Nakaji S, Fujii H, et al. Pseudoaneurysm caused by a self-expandable metal stent: a report of three cases. 
Endoscopy 2014;46:248-51.

6. Kamisawa T, Tu Y, Egawa N, et al. Histological study about metallic stent placed in biliary carcinoma after hyperthermo-chemo-radiation therapy. J Japan Biliary Assoc 1999;13:322-6.

7. Japan Pancreas Society. Clinical practice guidelines for pancreatic cancer 2019. Tokyo (Japan): Kanahara \& Co., Ltd., 2019.

8. Brierley J, Gospodarowicz MK, Wittekind C, editors. TNM classification of malignant tumours. 8th ed. Chichester (England): Wiley, 2017.

9. National Comprehensive Cancer Network. Clinical Practice Guidelines in Oncology: Pancreatic Adenocarcinoma. Version 1.2021. Criteria defining resectability status at diagnosis. 2020 October 23. Available online: https://www.nccn.org/professionals/physician_gls/ pdf/pancreatic.pdf

10. Cancer Therapy Evaluation Program. Common terminology criteria for adverse events (CTCAE) v5.0. US Department of Health and Human Services (US), 2017.

11. Eisenhauer EA, Therasse P, Bogaerts J, et al. New response evaluation criteria in solid tumours: revised RECIST guideline (version 1.1). Eur J Cancer 2009;45:228-47.

12. Kanda Y. Investigation of the freely available easy-touse software 'EZR' for medical statistics. Bone Marrow Transplant 2013;48:452-8.

13. Chauffert B, Mornex F, Bonnetain F, et al. Phase III trial comparing intensive induction chemoradiotherapy (60 Gy, infusional 5-FU and intermittent cisplatin) followed by maintenance gemcitabine with gemcitabine alone for locally advanced unresectable pancreatic cancer. Definitive results of the 2000-01 FFCD/SFRO study. Ann Oncol 2008;19:1592-9.

14. Park S, Park JY, Bang S, et al. Radiotherapy prolongs biliary metal stent patency in malignant pancreatobiliary obstructions. Gut Liver 2013;7:480-5.

15. Watanabe M, Shiozawa K, Mimura T, et al. Hepatic artery pseudoaneurysm after endoscopic biliary stenting for bile duct cancer. World J Radiol 2012;4:115-20.

16. Satoh T, Matsubayashi H, Ishiwatari H, et al. Rupture of Pseudoaneurysm after Biliary Metallic Stent Placement. Intern Med 2019;58:1453-7.

17. Park JY, Ryu H, Bang S, et al. Hepatic artery pseudoaneurysm associated with plastic biliary stent. Yonsei Med J 2007;48:546-8.

18. Yasuda M, Sato H, Koyama Y, et al. Late-onset severe biliary bleeding after endoscopic pigtail plastic stent insertion. World J Gastroenterol 2017;23:735-9.

19. Kim PH, Kim JW, Gwon DI, et al. Embolization for delayed arterial bleeding after percutaneous selfexpandable metallic stent placement in patients with malignant biliary obstruction. Br J Radiol 2020;93:20190637.

20. Isayama H, Yasuda I, Ryozawa S, et al. Results of a Japanese multicenter, randomized trial of endoscopic stenting for non-resectable pancreatic head cancer (JMtest): Covered Wallstent versus DoubleLayer stent. Dig Endosc 2011;23:310-5.

21. Lee H, Yoon J, Park K, et al. Dose perturbation by metallic biliary stent in external beam radiotherapy of pancreato-biliary cancers. Australas Phys Eng Sci Med 2019;42:745-56.
Cite this article as: Hayakawa S, Ito K, Hayakawa J, Murofushi KN, Karasawa K. Safety of biliary stent placement followed by definitive chemoradiotherapy in patients with pancreatic cancer with bile duct obstruction. J Gastrointest Oncol 2021;12(5):2260-2267. doi: 10.21037/jgo-21-198 